\title{
The Association between Cellular Phone Usage and Brain Neoplasms
}

\author{
Rohit Chander Chopra \\ Saba University School of Medicine, Saba, Netherlands \\ Email: rohitc89@gmail.com
}

How to cite this paper: Chopra, R.C. (2016) The Association between Cellular Phone Usage and Brain Neoplasms. Occupational Diseases and Environmental Medicine, 4, 103-115.

http://dx.doi.org/10.4236/odem.2016.44011

Received: July 26, 2016

Accepted: November 27, 2016

Published: November 30, 2016

Copyright $\odot 2016$ by author and Scientific Research Publishing Inc. This work is licensed under the Creative Commons Attribution International License (CC BY 4.0).

http://creativecommons.org/licenses/by/4.0/ (c) (i) Open Access

\begin{abstract}
Background: The association between cellular phones and brain tumors is a question that is frequently asked of the medical and scientific community. The prevalence of cell phone use and the significant morbidity and mortality of brain tumors contribute to this pairing. Cell phones are known to emit radio frequency energy in the form of both ionizing and non-ionizing radiation. Ionizing radiation is known to be within X-rays, which do have an association with cancer. Objective: To assess if the use of the cell phone has an association with brain tumors. Methods: The searches performed through PubMed were conducted to find studies that sought to provide evidence as to whether or not increased cell phone exposure contributed to the development of brain tumors. Also searched for was increased regional metabolism of the brain with the use of the cell phone switched in the on position. Studies were restricted to being published during or after the year 2000 and presented in the English language. Results: The studies largely support the conclusion that cell phone usage does not lead to the development of brain cancer. Studies employed different strategies, such as the prospective cohort and case-control studies to reach this conclusion. Both studies failed to show statistically significant evidence that cell phones were associated with brain tumors of the central nervous system. Conclusions: Questions raised by crossover studies demonstrating increased regional brain glucose metabolism continue to remain largely unanswered by current research and remain a starting point for future research. The prevalence of the issue strengthens its position among others as a matter that the medical community must continue to address to meet the needs of an increasingly exposed patient population. The overall hypothesis that cell phone usage does not lead to the development of brain tumors was supported.
\end{abstract}

\section{Keywords}

Cellular Phones, Mobile Phone, Brain Tumors, Cancer, Acoustic Schwannoma, Brain Glucose Metabolism, Radiofrequency Energy, Ionizing Radiation 


\section{Introduction}

The late 1990s oversaw a technological boom that sparked a rise in the prevalence of cellular phone usage in an adult population, and later teenagers, seeking to keep up with the direction and pace of society. The cell phone became a mainstay of modern society by rendering its predecessor-the pager-obsolete, and by infiltrating virtually all members of the household, with the exception of younger children.

By some estimates, the number of cell phone subscriptions worldwide reaches that of 4.6 billion, according to the International Telecommunications Union [1]. In the United States alone, the Cellular Telecommunications and Internet Association point out that the number of cell phone subscribers has "increased from 110 million in the year 2000 " to an astounding 303 million subscribers [2]. The reliance on the modern-day cell phone has only increased with the advent of today's smartphones. With these, users are able to enjoy much more than just Internet access, and more importantly, increase their cumulative exposure to the mobile devices themselves.

The concern lies within the fact that these devices emit radio frequency energy in the form of both ionizing and non-ionizing radiation. The latter of these is also found in microwaves and television-sets [2]. Ionizing radiation, however, has been linked to cancer in the past from different sources, such as from exposure to X-Rays. It is from these previous associations that the question arises about whether a credible link between cell phone usage and cancer can be established [3]. As an admission of this understanding, the United States Federal Communications Commission has placed restrictions on cellular phones sold within the United States to emit an acceptable maximum quantity of radiation to be absorbed by the body. This value, known as the Specific Absorption Rate (SAR), must be less than 1.6 watts per kilogram for the phone to be sold legally [4]. Even more concerning are the effects of radiation on the developing brain, especially with the "rise in cell phone popularity among children and adolescents in recent years." [3].

Society has historically paid the most attention to diseases that carry not just high morbidity, but also a high prevalence. While neurogenic tumors may not carry the prevalence of breast or lung cancer, it is well-established that metastases can occur secondary to either of these more prevalent neoplasms [5]. Unfortunately, it is an understatement to mention that both primary and secondary brain neoplasms carry significant mortality. Even following resection, mortality of the feared Glioblastoma Multiforme, the most common primary malignant neoplasm of the adult brain, remains high and carries a five-year survival rate of five percent [6]. With standard radiation and chemotherapy, the median survival time of patients with this specific neoplasm from the time of diagnosis is a disturbing fifteen months [7].

The nature of medicine relies on the assumption that if disease is preventable, the community will be given the necessary information about the means to prevent it from occurring. Therefore, if the risk factors for a given disease are deemed multifactorial in nature, an individual should be informed on how to decrease his or her chances to acquire the disease by controlling the environmental risk factors involved. 
The analysis of any potential links between cell phone usage and cancer are difficult to establish since there are genetic and environmental risk factors, allowing it to be deemed multifactorial. It is important to point out that healthcare providers especially seek those that are genetically predisposed to prevent the role that environmental risk factors play in the development of disease [8].

It is with this assumption that a potential patient entertaining the discussion of risk factors may ask the question, "Does my usage of this cell phone increase my chances of getting a brain tumor in the future?" The responsibility of the physician is to provide an informed response in a manner that allows the patient to understand how emitted radio frequency energy affects the body with respect to current research.

The sheer prevalence of cell phone usage makes this a global question that modern physicians cannot ignore. The fact that research concerning the issue is conducted on a regular basis illustrates its significance in today's society.

\section{Methods}

In the process of collecting data for this review, certain restrictions were placed for the search and selection of the vast number of articles available for usage. The primary criteria for selection included both pertinent and current research. Although the advent of the cellular phone can be attributed to the 1980s, no studies published prior to the year 2000 were considered in this discussion. However, background information from earlier articles was still considered valuable to understanding the developments going on at the time.

PubMed (http://www.ncbi.nlm.nih.gov/pubmed) was the primary database utilized to search for relevant studies. Keywords searched included combinations of the following MeSH terms: Cellular Phones AND Central Nervous System Tumors, Mobile Phones AND Brain Cancer, Cell Phones AND Radio Frequency Emissions, Cell Phones AND Brain Glucose Metabolism. Exceptions to these search terms included the analysis of cell phone usage causing any central nervous system disease. However, these publications must have included specific data regarding tumors of the central nervous system to be considered for discussion.

The types of articles were restricted to prospective cohort, case-control and crossover studies. An additional search was performed on the National Cancer Institute (www.cancer.gov), with a search for "cell phone usage" statistics in the United States used in the introduction. Search criteria were limited to those articles available in the English language.

\section{Results}

\subsection{American Cell Phone Usage in Relation to Brain Tumor Incidence Trends}

A relatively simple method to explore a possible association between cell phone usage and brain tumors is to monitor the incidence of brain tumors over the period of time that cell phones infiltrated society. The 2010 publication under Inskip et al. did just this 
by taking data from the Surveillance Epidemiology and End Results (SEER) Program about brain tumor incidence and analyzing trends from the years 1992 to 2006 among whites. The study then went further by analyzing incidences among different age groups and by gender, as well as among different types and locations of brain tumors. The study decided to exclude results from leukemias, lymphomas and meningiomas. The publication used these age-adjusted and age-specific incidence rates to establish a log-linear model standardized to the population of the United States in the year 2000. Using the statistical method under Tiwari et al., this log-linear model was then used to evaluate the annual percent change of the incidence rate [9]. The study found that the overall incidence of brain tumors has seen a decline in the United States among all age groups, with the exception of women ages 20 to 29, from the years 1992 to 2006 . The study investigated the isolated increase in incidence among younger women and found it to be predominately cancers within the frontal lobe, and not in the parietal or temporal location suspected with cell phone usage. Overall, the study found that the incidence data analyzed yielded no support to the view that brain tumors are caused by cell phone usage. They found that the data related to the rise in frontal lobe cancers was shown to be "inconsistent with a causal interpretation" from cell phones [10].

\subsection{Increased Regional Glucose Metabolism}

A unique development in new research regarding the possible association between cell phone usage and brain tumors is that of the role of the brain's glucose metabolism. The 2011 publication under Volkow et al. describes a relatively clinical approach to the question that has largely been studied epidemiologically. The researchers began the experiment by initiating ongoing measurements of brain glucose metabolism by using Positive Electron Tomography. The experiment continued by having volunteers use cell phones with the phone switch in the on position for a set period of time and then comparing brain glucose metabolism values to that of the individual's previously-recorded baseline values. The results of the experiment were able to demonstrate that the glucose metabolism of the region of the brain closest to the antenna had a mode of 35.7 $\mu \mathrm{mol} / 100 \mathrm{~g}$ per minute, as opposed to 33.3 with the cell phone turned off (P-value: 0.004). The average difference was found to be 2.4 ( $95 \%$ CI 0.67 to 4.2 ) [11]. The results were also able to demonstrate that the region of the brain with increased brain glucose metabolism was closest to the antenna and developed models and illustrations accordingly. It remained clear from the crossover study that the increased brain glucose metabolism resulted from cell phone usage [11]. It is important to note that the subjects in this study were not talking while the cell phone was placed next to their ears, regardless of whether the cell phone was switched on or off.

\subsection{Cohort Studies Investigating Neurogenic Tumor Incidence among Cell-Phone Subscribers}

A reasonable procedure to evaluate the effects of radio frequency exposure from cell phones on humans is to both calculate the incidence of the suspected disease, and to 
understand the temporal sequence from the beginning of the exposure to the outcome. To achieve this, the prospective cohort study serves as the optimal tool for the assessment of multiple outcomes, or in this case, different types of brain tumors. In 1987, a group of researchers under Frei et al. sought to implement a cohort study among Danish cell phone subscribers by tracking the incidence of cancer among them up until the year 2007. The results were then divided by gender, the variable of smoking, and interestingly those that had greater than twelve years of education. For each group, the results were subdivided into cancers of the central nervous system, which were then incorporated into table used for analysis. The resulting incidences established that there existed no statistical significance among either group when the confounding factor of smoking was taken out of the equation. According to the study, each calculated incidence for each subgroup was close to unity (approximately close to 1) or decreased (below 1), including for those with over 13 years of cell phone subscription. Specifically for tumors of the central nervous system, the study found that there was no overall increased risk, a decreased risk of meningioma, and for men a non-significant increased risk for glioma in men. This study found that in long-term subscribers, "there was no increased risk of glioma in temporal lobe" (incidence rate ratio of 1.13; CI: 0.86 - 1.48) [12], a concern which had been suggested by the analysis of the experiment studying the regionally increased brain glucose metabolism by Volkow et al. [11].

Similar to the aforementioned Danish cohort study by Frei et al., a prospective cohort study by Benson et al. in 2013 sought to assess whether or not there existed an increased risk of intracranial central nervous system tumors, as well as other cancers among cell phone subscribers by using existing data. The study collected data from 1999 to 2005 and subdivided each group by gender, just as the previously mentioned study had also done. This data recorded those who developed disease and tracked the amount of day-to-day cell phone use as well as the duration of use in years. The resulting data were subsequently utilized to assess the relative risks, which were subdivided by the type of cancer as well. Specifically to intracranial neoplasms of the central nervous system, the study found that the relative risk of ever using a cell phone in such patients was not statistically significant at a relative risk of 1.01 (95\% CI: 0.90 to 1.14) [13].

\subsection{Case-Control Studies and Animal Models Examining Brain Tumors from Radiation Exposure}

A logical approach to explore a possible association between brain tumors and radiation exposure in a short amount of time would be to analyze existing data from a casecontrol study. The publication by Hardell et al., did just that by employing data from pooled case-control studies about patients both living and deceased that were diagnosed with a neoplasm of the central nervous system between the years 1997-2003. Using this data, the study then directed its goal at finding out whether or not these groups of patients were exposed to both cellular and cordless phones. To do this, the research group interviewed living patients and relatives of the deceased by using structured questionnaires to find out their cumulative exposure in number of hours. The resulting 
odds ratio regarding patients with gliomas, meningiomas, and all malignant tumors were incorporated into a table for analysis. For those with over 74 hours of cumulative mobile phone exposure, the study found a statistically significant increased risk for all malignant tumors past a ten-year latency (OR: 2.7; CI: 1.9 - 3.7) [14]. As opposed to the aforementioned cohort studies, the study by Hardell et al. found that there existed a statistically significant increased risk for glioma in patients with cumulative exposure to mobile phones greater than 74 hours [14].

The long-term effects of cellular phone usage on the developing brain became a grave concern for potential subscribers early in its introduction. The publication under Aydin et al. sought to address this potential problem by implementing a multi-center casecontrol study on children and adolescents residing within Denmark, Norway, Sweden, and Switzerland. This study used data from individuals from the ages 7 to 19 that were diagnosed with a neurogenic tumor from the years 2004 to 2008. Similar to the publication under Hardell et al., the study used the data from these patients to ascertain whether or not they were exposed to mobile phones. The study then sought to find out the cumulative amount of exposure in hours, the cumulative duration of active cell phone subscription in years, and also the time since the first use of the cell phone in years. Again similar to Hardell et al., the publication then used this data to calculate odds ratios to assess any risk mobile phone usage plays on the development of brain tumors. Furthermore, the researchers were keen to point out the potential impact of confounding variables, such as cordless phone usage and birth weight, may have in the analysis. As a result of this potential impact, the researchers were open to making any adjustments to developed models accordingly if the calculated odds ratio changed by ten percent or more under its influence. In fact, because the resultant changes on risk estimates failed to reach ten percent, none of the confounders were included in the conditional logistic regression models presented in the publication. The study found that when compared to the subjects of the control group, regular mobile phone users were not at a statistically significant increased risk of being diagnosed with a brain tumor (OR: 1.36; 95\% CI 0.92 to 2.02) [15]. The publication also found that children who began using cell phones over five years earlier were not at a statistically significant increased risk "compared with those who never regularly used mobile phones (OR: 1.26; 95\% CI 0.70 to 2.28)." [15].

A third case-control study under Muscat et al. sought to take a much more general approach in comparison to the previously mentioned studies. The researchers used data from American patients diagnosed with a primary brain tumor between the years 1994 and 1998 to assess the risk from cell phone radiation exposure in the development of their disease. These cases were matched to controls that were not diagnosed with a primary brain tumor. The researchers then used structured questionnaires to ask the individuals to quantify their cell phone exposure in hours per month as well as in years of usage. The researchers subsequently used this data to separate them into groups based on the amount and duration of exposure. Based on the data, the publication found that for those that used a cell phone less than 0.72 hours per month, the overall risk for a primary brain tumor was close to unity (OR: 1.0; 95\% CI 0.5 to 2.0) [16]. The 
study also found no association between frequent cell phone users (greater than 10.1 hours per month) and primary brain tumors (OR: 0.7 ; 95\% CI 0.3 to 1.4) [16]. According to the study, no association was demonstrated between duration of cell phone use in years and primary brain tumors (P-value: 0.54). It is important to note that the researchers were keen to point out that for the primary brain tumors that take years to develop, further research would be needed to rule out an association with cellular phone usage.

To respond to this call for new research on slow-growing tumors by the previously mentioned study under Muscat et al, the case-control study under Lönn et al. sought to answer that question. It is understood that a more specific concern regarding cell phones pertains to radiofrequency emissions concentrated at the area closest to the device, which includes the auditory nerve. From this information, one makes the understandable deduction that any risk of acoustic neuroma must be investigated before it becomes a widespread problem for the public. The Swedish publication under Lönn et al. worked to examine the risk posed by radio frequency from cellular phones towards the development of acoustic neuromas. Central to the analysis of the paper was a casecontrol study conducted by the researchers, in which the cases were patients from the ages 20 to 69 and were diagnosed with acoustic neuromas between the years 1999-2002. Both cases and controls were asked in detail about their exposure to mobile phone use, particularly the side of the head to which the individual uses a cell phone most often. Results were adjusted to take into account confounding variables, such as age, gender, and whether hearing loss was exhibited five years prior to diagnosis for those with acoustic neuroma.

It was important to note that radiofrequency exposure is viewed as higher for rural areas because of the increased output power level needed to for the device to communicate with its base station. For this reason, analysis for the variable of whether the individual resides in a rural or an urban residence was considered separately. The study found that the overall risk for acoustic neuroma with regular cell phone use was close to unity (OR 1.0; CI 0.6 to 1.5) [17]. The study then considered those diagnosed with acoustic neuromas ten years after the initiation of cell phone use, for which the overall risk was increased (OR 1.9; CI 0.9 to 4.1) [17]. The study then divided the cases into two groups depending on whether the disease presented on the right or left side of the head. For ipsilateral tumors, which are seen on the same side as the individual's predominant cell phone use, the risk was much higher (OR 3.9; CI 1.6 to 9.5) [17]. Conversely, the risk for tumors contralateral to the side of predominant cell phone use was understandably much lower (OR 0.8; CI 0.2 to 2.9).

Another pertinent concern regarding cellular phone usage is specific to the high frequency radio frequency emissions that are understood to accompany the conveniences of device usage. The goal of the publication by Zook et al., was to use animal-models to examine the effects of radio frequency emissions on ethylnitrosourea-induced neurogenic tumors. The study emulated cell phone radio frequency emissions by setting a pulsed exposure at $860 \mathrm{MHz}$ onto Sprague-Dawley rats. The radio frequency (RF) ex- 
posure was regularly administered at 6 hours per day for 5 days per week. The tumors were induced using either 6.25 or $10 \mathrm{mg} / \mathrm{kg}$ ethylnitrosourea (ENU), which is known to induce neurogenic tumors onto the Sprague-Dawley species of rat [18]. The researchers then recorded the incidence of tumors in the rats to ascertain a possible shortened latency time that could be attributed to the pulsed radiofrequency signal. However, given that the tumor incidence in radio frequency-exposed rats was actually slightly lower than that of the control group, the study was unable to point out any effect of the radio frequency exposure on the development of the tumor [18].

The study left to discussion the possibility of a promotional effect by the radio frequency exposure on a shortened latency of tumor growth. It is important to note however, that the study was keen to point out that the results of this shortened latency were deemed statistically insignificant. The tumors were analyzed for its histological characteristics as well, and were deemed to show no evidence that radio frequency exposure had any effect on the "incidence, malignancy, volume, multiplicity, latency, or fatality associated with any kind of neurogenic tumor." [18].

\section{Discussion}

The studies mentioned in this discussion employ extraordinarily different strategies to support the consensus that researchers have established over the past thirty years since the advent of the mobile phone. The Danish cohort study by Frei et al., establishes there exists no statistical significance to assert that mobile phones cause cancer of the central nervous system [13]. The study goes further to effectively respond to the question raised by a previously discussed crossover study by Volkow et al., of whether a regional increase in brain glucose metabolism should raise concerns for cancer or not [11]. It makes sense for one to deduce that the specific cancer implied would be limited to the region discussed in the study, particular of a temporal lobe glioma.

The study by Frei et al. maintained that there was no statistical significance among Danish cell phone subscribers to support such an assertion that these types of tumors were at an increased incidence due to cell phone usage [12]. Finally, a prospective study by Benson et al., collected data among today's cancer patients (2013) to achieve similar conclusions as the Danish cohort study, that there was no evidence to support an increased risk of cancer among those that ever used a cell phone, claimed to use the device daily, or for an increased duration over ten years [13].

A significant point of contention remains from the study under Hardell et al., which found that there existed an increased risk of glioma from a cumulative exposure to mobile phones greater than 74 hours [14]. Upon further analysis of this study and of publications with similar objectives, significant limitations existed in its execution. A study under Ahlbom et al., had the specific objective to review and pool data from Hardell et al. with similar publications. The publication was keen to point out that the study under Hardell et al. was prone to a selection bias under non-responders from the existing data [19]. Ahlbom et al. argues that an analysis of the odds ratio of 2.7 calculated by the study shows it to be an outlier among closely related publications, which was shown in 
the figure used for analysis [19]. Pooled estimates of the odds ratios calculated by the other studies with similar objectives to Hardell et al., were shown to be closer to unity when that of Hardell et al. was not taken into account.

Furthermore, apart from the review by Ahlbom et al, the nature of the data from the pooled case-controls by Hardell et al. allows for confounding variables unaccounted for in its presented argument to cast questions on the certainty of its analysis. These confounding variables range from hereditary predispositions to other environmental exposures other than mobile phone use that patients were not asked about. A final limitation to the study by Hardell et al. is that of recall bias, as it is inherently difficult for patients to remember the specific number of lifetime hours of mobile phone usage. These limitations were not addressed in the study, but were important in the analysis of the research and may have contributed to its calculated odds ratio being an outlier [19].

The publications presented by both Hardell et al. and Aydin et al. both featured casecontrol studies to reach their analyses. The fact that the case-control study under Aydin et al. decided to take into account confounding variables is the key distinction that makes its conclusions much stronger than of those presented under Hardell et al. Although it is an understatement to claim that the conclusions reached by the latter are concerning, its lack of mention of potential confounders and questionable methodology cast doubt on its research as a whole [20]. The analysis under Aydin et al. serves to fortify the consensus that cell phones do not cause brain tumors, as does the doubt surrounding the weaker publication under Hardell et al., which argues the contrary.

The publication under Inskip et al. offered a unique incidence analysis of brain tumors in the United States throughout the years attributed to the rise of the cell phone. Although it offered a clear analysis to reinforce its assertion that cell phones are not associated with brain tumors, the study acknowledged a number of limitations to its methodology. By nature of the set up of the study, trends from tumors with a very long induction period would take many years to be seen in general population data. The study admitted that although it may be too soon for analysis of these tumors, "even for a long mean induction time, one would expect a distribution around this mean." [10]. The article also acknowledged that SEER did not account for benign intracranial tumors such as meningioma and acoustic neuroma in the original population data [10]. Combined with the fact that it would take longer to account for slow-growing tumors, a growing theme among researchers is that tumors such as acoustic neuromas remain elusive targets for analysis. This sentiment was shared by researchers under the publication by Muscat et al, who viewed it as a limitation to their study, as well as by those under Lönn et al, whose primary objective to overcome it [17].

The American case-control study under Muscat et al. served to reinforce the growing evidence at the time against cell phone usage causing brain tumors. However, the article was open to pointing out that there remained limitations in its analysis that needed to be ironed out past the time of publication. A notable example of this was the admission that brain tumors with longer induction periods, such as that of acoustic neuroma, were not accounted for in the research [16]. Secondly, the publication also indicated 
that its data did not take into account any tumorigenic potential posed by cell phone radio frequency to act as a promoter [16]. Although the previously mentioned study under Zook et al. did its best to respond to this concern by noting changes to tumor histology within animal models, the researchers under Muscat et al. did not perform brain biopsies on their cases. In spite of the fact that it can be argued that this can be seen as a limitation in the article's analysis, it is for understandably obvious reasons of research ethics that these histopathological analyses were not carried out on their patients.

As much evidence has been presented demonstrating that cell phones do not cause brain tumors, the case-control study under Lönn et al. has shown itself to be one of few publications that argue in favor of cell phones causing a specific brain tumor while addressing issues that could weaken their analysis. The article does take into account the effects of potential confounding variables, recall bias among individuals about their past cell phone usage, as well as selection bias introduced by high non-response rates among controls. The article asserts that long-term cell phone use greater than ten years increases the risk for acoustic neuroma on the same side as the individual's predominant cell phone use [17]. However, the analysis under Lönn et al. was criticized by the researchers' colleagues because acoustic neuroma is understood to be a tumor that develops very slowly. Therefore, the "only etiologically viable analysis would be among those who were exposed years before diagnosis." [21].

The publication under Lönn et al. was praised as the article that stood alone demonstrating viable evidence showing a long-term association between cell phones and an ipsilateral acoustic neuroma [20]. The research group's colleagues constructively criticized its methodology, exposing a detection bias among cases who were more likely to be diagnosed for an acoustic neuroma by reporting its first symptom: ipsilateral hearing loss [20]. The resultant detection bias was argued to contribute to a positive association for acoustic neuromas of the affected side and hence, an inflated odds ratio not accounted for by the original publication [20].

The concerns regarding the effects of radio frequency exposure remain related to questions posed by the increased brain glucose metabolism demonstrated in the crossover study by Volkow et al. [11]. Zook et al. performed histopathological analysis to discover that there was no evidence to determine that there were significant effects of radio frequency exposure on neurogenic tumor incidence [18]. A limitation to the study to be considered is that animals, in this case, rats, are different in their molecular biology from humans.

While the effects of this difference in analysis of the resultant data is difficult to determine, it is important to note that the radio frequency employed in the article was different than that of many cellular phones. According to the publication by Zook et al. itself, many cellular phones emit a radio frequency of approximately $2450 \mathrm{MHz}$. The article however elected to subjugate the rats to an exposure of the $860 \mathrm{MHz}$ as mentioned previously [18]. The effects of this difference in radio frequency have the potential to be highly significant, and the article was keen to point out that other publications have called for future research to address this concern [18]. 
As confident as each study was in their respective results, the study by Frei et al. thought it important to point out that there existed limitations within its study. For example, the study stated that it was unable to decipher who amongst the subjects can be categorized as exposed to a high versus a low degree of cell phone use [12]. The prospective study by Benson et al. attempted its best at resolving this issue by categorizing its subjects as users that had used a cell phone at least once, daily users, or users that can claim having an exposure greater than ten years [13].

The crossover study on increased regional brain glucose metabolism by Volkow et al. did pose a question, which was provided an answer at least somewhat by the Danish crossover study by Frei et al. This answer, however, was only provided for one type of cancer that can occur in the region of the temporal lobe: gliomas. The question of what the significance of an increased regional brain glucose metabolism in the proximity of a cell phone antennae can only be answered with more research.

\section{Conclusions}

The broad question posed by the international community about whether cell phone usage leads to an increased risk for brain cancers is a pertinent and prevalent question that will not go away on its own. In this modern age, virtually everyone in the developed world, from adults to even some young children, is exposed to the cellular phone at some point in their lives. It is therefore a legitimate concern for a potential patient to bring up to his or her physician on the risks that come with the cell phone in hand. Physicians have the duty of being informed about both the established consensus and developing research within the medical community. When both are combined, clinicians should be able to provide an answer for the patient to understand the risks of the discussed environmental exposure.

The articles presented in this discussion share a common theme in their analysis: further research is essential to answer a changing question. Older research deemed valuable for background information on the issue does not take into account today's generation of smartphones. Computers are now making way for the next generation of "phone-tablets," which are phones that carry many of the same functions as a computer. What this means for newer users of cellphones is that their cumulative exposure to high frequency radiation may now be even greater than ever before. Future research can be as simple as repeating an older experiment regarding the higher radio frequency exposure on rats and changing its radio frequency to $2450 \mathrm{MHz}$. Newer research can also be different as to attempt an answer at the complex set of questions posed by the increased regional brain glucose metabolism shown by Volkow et al.

The majority of relevant research establishes that cell phone usage does not lead to the development of brain cancer. Despite this understanding, crossover studies that demonstrate an increased regional brain glucose metabolism have kept researchers working to keep the medical community informed regarding the latest developments on the issue. The sheer prevalence of brain cancer and on a much, much larger scale the use of cellular phones, make this an issue that will continue to need further research. 


\section{References}

[1] (2010) International Telecommunication Union: Measuring the Information Society. http://www.itu.int/ITU-D/ict/publications/idi/material/2010/MIS 2010 without annex 4e.pdf

[2] National Cancer Institute (2013) Cell Phones and Cancer Risk. United States. http://www.cancer.gov/cancertopics/factsheet/Risk/cellphones

[3] National Cancer Institute (2010) Surveillance Epidemiology and End Results (SEER) Data. http://seer.cancer.gov/

[4] Federal Communications Commission (2014) Specific Absorption Rate (SAR) for Cell Phones: What It Means for You.

http://www.fcc.gov/guides/specific-absorption-rate-sar-cell-phones-what-it-means-you

[5] National Cancer Institute (2014) General Information about Adult Brain Tumors. http://www.cancer.gov/cancertopics/pdq/treatment/adultbrain/Patient/page1/AllPages

[6] Central Brain Tumor Registry of the United States (2011) CBTRUS Statistical Report: Primary Brain and Central Nervous System Tumors Diagnosed in the United States in 20042007. http://www.cbtrus.org/2011-NPCR-SEER/WEB-0407-Report-3-3-2011.pdf

[7] Johnson, D.R. and O’Neill, B.P. (2011) Glioblastoma Survival in the United States before and during the Temozolomide Era. Journal of Neuro-Oncology, 107, 359-364. http://dx.doi.org/10.1007/s11060-011-0749-4

[8] International Agency for Research on Cancer (2014) IARC Monographs on the Evaluation of Carcinogenic Risks to Humans. http://monographs.iarc.fr/ENG/Classification/ClassificationsAlphaOrder.pdf

[9] Tiwari, R.C., Clegg, L.X. and Zou, Z. (2006) Efficient Interval Estimation for Age-Adjusted Cancer Rates. Statistical Methods in Medical Research, 15, 547-569.

http://dx.doi.org/10.1177/0962280206070621

[10] Inskip, P.D., Hoover, R.N. and Devesa, S.S. (2010) Brain Cancer Incidence Trends in Relation to Cellular Telephone Use in the United States. Neuro-Oncology, 12, 1147-1151. http://dx.doi.org/10.1093/neuonc/noq077

[11] Volkow, N.D., Tomasi, D., Wang, G.-J., Vaska, P., Fowler, J.S., Telang, F., Alexoff, D., Logan, J. and Wong, C. (2011) Effects of Cell Phone Radiofrequency Signal Exposure on Brain Glucose Metabolism. The Journal of the American Medical Association, 305, 808-813. http://dx.doi.org/10.1001/jama.2011.186

[12] Frei, P., Poulsen, A.H., Johansen, C., et al. (2011) Use of Mobile Phones and Risk of Brain Tumours: Update of Danish Cohort Study. British Medical Journal, 343, d6387. http://dx.doi.org/10.1136/bmj.d6387

[13] Benson, V.S., Pirie, K., Schüz, J., et al. (2013) Mobile Phone Use and Risk of Brain Neoplasms and Other Cancers: Prospective Study. International Journal of Epidemiology, 1-11.

[14] Hardell, L., Carlberg, M. and Hansson Mild, K. (2011) Pooled Analysis of Case-Control Studies on Malignant Brain Tumours and the Use of Mobile and Cordless Phones Including Living and Deceased Subjects. International Journal of Oncology, 38, 1465-1474. http://dx.doi.org/10.3892/ijo.2011.947

[15] Aydin, D., Feychting, M., Schuz, J., et al. (2011) Mobile Phone Use and Brain Tumors in Children and Adolescents: A Multicenter Case-Control Study (CEFALO). Journal of the National Cancer Institute, 103, 1264-1276. http://dx.doi.org/10.1093/jnci/djr244

[16] Muscat, J.E., Malkin, M.G., Thompson, S., Shore, R.E., Stellman, S.D., McRee, D., Neugut, A. and Wynder, E. (2000) Handheld Cellular Telephone Use and Risk of Brain Cancer. The 
Journal of the American Medical Association, 284, 3001-3007.

http://dx.doi.org/10.1001/jama.284.23.3001

[17] Lönn, S., Ahlbom, A., Hall, P. and Feychting, M. (2004) Mobile Phone Use and the Risk of Acoustic Neuroma. Epidemiology, 15, 653-659.

http://dx.doi.org/10.1097/01.ede.0000142519.00772.bf

[18] Zook, B.C. and Simmens, S.J. (2006) The Effects of Pulsed $860 \mathrm{MHz}$ Radiofrequency Radiation on the Promotion of Neurogenic Tumors in Rats. Radiation Research, 165, 608-615. http://dx.doi.org/10.1667/RR3551.1

[19] Ahlbom, A., Feychting, A., Kheifets, L., Savitz, D. and Swerdlow, A. (2009) Epidemiologic Evidence on Mobile Phones and Tumor Risk. Epidemiology, 20, 639-652. http://dx.doi.org/10.1097/EDE.0b013e3181b0927d

[20] Savitz, D. (2004) Mixed Signals on Cell Phones and Cancer. Epidemiology, 15, 651-652. http://dx.doi.org/10.1097/01.ede.0000142650.10465.97

[21] Stang, A. and Jockel, K. (2005) Mobile Phone Use and Acoustic Neuromas. Epidemiology, 16, 414-415. http://dx.doi.org/10.1097/01.ede.0000158819.64085.b2

Submit or recommend next manuscript to SCIRP and we will provide best service for you:

Accepting pre-submission inquiries through Email, Facebook, LinkedIn, Twitter, etc. A wide selection of journals (inclusive of 9 subjects, more than 200 journals)

Providing 24-hour high-quality service

User-friendly online submission system

Fair and swift peer-review system

Efficient typesetting and proofreading procedure

Display of the result of downloads and visits, as well as the number of cited articles Maximum dissemination of your research work

Submit your manuscript at: http://papersubmission.scirp.org/

Or contact odem@scirp.org 